\title{
Primeiro registro de Evandromyia (Aldamyia) sericea (Floch \& Abonnenc) (Diptera, Psychodidae, Phlebotominae) para a região Sudeste do Brasil
}

\author{
Israel de Souza Pinto ${ }^{1,2}$, Claudiney Biral dos $\operatorname{Santos}^{1}$, Adelson Luiz Ferreira ${ }^{1} \&$ Aloísio Falqueto ${ }^{1}$
}

\begin{abstract}
${ }^{1}$ Unidade de Medicina Tropical, Universidade Federal do Espírito Santo, Av. Marechal Campos 1468, 29040-090 Vitória-ES, Brasil. ${ }^{2}$ Programa de Pós-Graduação em Biologia Animal, Universidade Federal do Espírito Santo, Av. Marechal Campos 1468, 29043-900 VitóriaES, Brasil. Bolsista Capes. pintoisrael@gmail.com
\end{abstract}

\begin{abstract}
First record of the Evandromyia (Aldamyia) sericea (Floch \& Abonnenc) (Diptera, Psychodidae, Phlebotominae) in southeastern Brazil. This is the first record of Evandromyia (Aldamyia) sericea in southeastern Brazil and report the southernmost record ever of this species in the world. Specimens were collected in the Reserva Biológica de Duas Bocas, municipality of Cariacica, state of the Espírito Santo. Captures of the sand flies were carried out monthly from April to June 2008 during four consecutives nights using two automatic light traps (CDC model) and a modified Shannon trap for each night. The finding of this species corroborates the hypothesis of the phylogeographical proximity of the Amazon Rainforest and the Atlantic Forest.
\end{abstract}

KEYWORDS. Atlantic Forest; geographical distribution; sand fly.

RESUMO. Primeiro registro de Evandromyia (Aldamyia) sericea (Floch \& Abonnenc) (Diptera, Psychodidae, Phlebotominae) para a região Sudeste do Brasil. Esse é o primeiro registro de Evandromyia (Aldamyia) sericea para o Sudeste do Brasil e, até então, o registro mais ao sul da espécie no mundo. Os espécimes foram coletados na Reserva Biológica de Duas Bocas, município de Cariacica, estado do Espírito Santo. As capturas de flebotomíneos foram feitas mensalmente de abril a junho de 2008 durante quatro noites consecutivas usando duas armadilhas automáticas do tipo CDC e uma armadilha de Shannon modificada para cada noite. O encontro dessa espécie é consistente com a hipótese de proximidade filogeográfica entre a Floresta Amazônica e a Mata Atlântica.

PALAVRAS-CHAVE. Distribuição geográfica; flebotomíneo; Mata Atlântica.

O conhecimento sobre a distribuição geográfica dos flebotomíneos (Diptera, Psychodidae, Phlebotominae) é fundamental para entender fatores relacionados à transmissão de agentes etiológicos de algumas doenças, principalmente as leishmanioses. Essa distribuição é influenciada por fatores ecológicos como vegetação, altitude, relevo e clima (GebreMichael et al. 2004; Peterson \& Shaw 2003).

A região Sudeste do Brasil é composta pelos estados do Espírito Santo, Minas Gerais, Rio de Janeiro e São Paulo e apresenta registros de 109 espécies de flebotomíneos pertencentes aos gêneros: Bichromomyia Artemiev, 1991; Brumptomyia França \& Parrot, 1921; Deanemyia Galati, 1995; Evandromyia Mangabeira, 1941; Expapillata Galati, 1995; Lutzomyia França, 1924; Martinsmyia Galati, 1995; Micropygomyia Barretto, 1962; Migonemyia Galati, 1995; Nyssomyia Barretto, 1962; Pintomyia Costa Lima, 1932; Pressatia Mangabeira, 1942; Psathyromyia Barretto, 1962; Psychodopygus Mangabeira, 1941; Sciopemyia Barretto, 1962 e Trichopygomyia Barretto, 1962. Para o gênero Evandromyia, são registradas 14 espécies: E. (Aldamyia) evandroi (Costa Lima \& Antunes, 1936), E. (A.) lenti (Mangabeira, 1938), E. (A.) termithophila (Martins, Falcão, \& Silva, 1964), E. (A.) walkeri (Newstead, 1914), E. (Barrettomyia) teratodes (Martins, Falcão \& Silva, 1964), E. (B.) callipyga (Martins \& Silva, 1965), E. (B.) cortelezzii (Brèthes, 1923), E. (B.) costalimai (Mangabeira, 1942), E. (B.) petropolitana (Martins \& Silva, 1968), E. (B.) tupynambai (Mangabeira, 1942), E. (B.) edwardsi (Mangabeira, 1941), E. (B.) sallesi (Galvão \& Coutinho, 1939), E. (Evandromyia) bourrouli (Barreto \& Coutinho, 1941), E. (E.) rupicola (Martins, Godoy \& Silva, 1962) (Martins et al.1978; Young \& Duncan 1994; Aguiar \& Medeiros 2003; Galati 2003a; Galati et al. 2003; Pinto \& Santos 2007; Shimabukuro et al. 2007).

No estado do Espírito Santo foram assinaladas 57 espécies e não se tem registro do gênero Deanemyia. Para o gênero Evandromyia, não há registros de cinco espécies: $E$. (A.) walkeri, E. (B.) teratodes, E. (B.) cortelezzii, E. (E.) bourrouli e E. (E.) rupicola (Martins et al.1978; Young \& Duncan 1994; Ferreira et al. 2001; Aguiar \& Medeiros 2003; Galati 2003a; Pinto \& Santos 2007; Pinto et al. 2008).

Evandromyia (Aldamyia) sericea (Floch \& Abonnenc, 1944), descrita a partir de exemplares coletados na Guiana 
Francesa, tem sido registrada também no Brasil, Equador, Suriname e Venezuela. No Brasil, foi encontrada em estados das regiões Centro-Oeste (Mato Grosso), Nordeste (Bahia e Ceará) e Norte (Acre, Amapá, Amazonas, Pará, Rondônia e Roraima) (Young \& Duncan 1994; Aguiar \& Medeiros 2003; Galati 2003a). Aguiar \& Medeiros (2003) verificaram que esse flebotomíneo apresenta hábitos silvestres, sendo encontrado somente em ambientes florestais. A espécie ainda não foi associada à transmissão de Leishmania, não havendo registro de infecção natural por formas flageladas (Freitas et al. 2002).

O presente trabalho tem por objetivo acrescentar E. (A.) sericea à fauna flebotomínica conhecida da região Sudeste do Brasil.

Os exemplares de E. (A.) sericea foram coletados na Reserva Biológica Duas Bocas (20 18' 05" S e 40²8'06” W), município de Cariacica, estado do Espírito Santo, Brasil. A área é coberta por Mata Atlântica de encosta e apresenta relevo acidentado com altitudes variando de 450 a $850 \mathrm{~m}$ acima do nível do mar. O solo da região é classificado como latossolo vermelho-amarelo distrófico com textura argilosa e acentuadamente drenado. É uma região chuvosa (déficit hídrico anual de 56 a $174 \mathrm{~mm})$ e de temperaturas amenas (11,8 a $9,4^{\circ} \mathrm{C}$ de média das mínimas no mês mais frio e 30,7 a $27,8^{\circ} \mathrm{C}$ de média das máximas no mês mais quente) (Feitoza 1999).

Foram realizadas campanhas mensais no período de abril a junho de 2008, durante quatro dias consecutivos. Em cada dia, foram utilizadas uma armadilha de Shannon modificada, na qual os flebotomíneos eram coletados com capturador de sucção do tipo Castro, entre 18:00 e 21:00 horas, e duas armadilhas automáticas luminosas, tipo CDC (Center for Disease Control) entre 18:00 e 06:00 horas.

Os flebotomíneos coletados foram montados em lâminas, segundo Barretto e Coutinho (1940) e identificados segundo critérios taxonômicos propostos por Galati (1995, 2003 b). Espécimes testemunho foram depositados na coleção do Centro de Referência Nacional e Internacional para Phlebotominae do Centro de Pesquisa René Rachou FIOCRUZ, Belo Horizonte, Brasil.

Foram coletados sete exemplares de E. (A.) sericea, sendo dois machos e cinco fêmeas. Com esse encontro, são 110 espécies de flebotomíneos registradas na região Sudeste do Brasil, das quais 15 pertencem ao gênero Evandromyia. No estado do Espírito Santo, agora, são registradas 58 espécies de flebotomíneos e 10 para esse gênero.

$\mathrm{Na}$ região Sudeste, as coletas de flebotomíneos são realizadas principalmente em locais em que ocorreram casos de leishmanioses, representados por áreas modificadas pela ação humana com presença de vetores adaptados a esse ambiente e de reservatórios domésticos das leishmânias (e.g., Barros et al. 1985; Falqueto et al. 1986). Os ambientes florestais preservados são pouco explorados e a composição da fauna de flebotomíneos silvestres é pouco conhecida, justificando em parte o registro tardio de $E$. (A.) sericea para a região. Soma-se a isso o fato de que em diversos estudos a quantidade de exemplares dessa espécie é muito baixa quando comparada à de outros flebotomíneos silvestres, mesmo na região
Amazônica, onde é coletada com freqüência (Freitas et al. 2002). Isso também justifica o registro tardio da espécie na região Sudeste.

E. (A.) sericea parece não ocorrer na região central do Brasil, onde o bioma é o Cerrado, mas é encontrada em áreas de Floresta Amazônica e de Mata Atlântica. Isso corrobora os estudos filogeográficos de Costa (2003), nos quais conclui que espécies de pequenos mamíferos de áreas de floresta Amazônica e de Mata Atlântica são mais próximas filogeograficamente entre si do que de espécies de áreas florestais da região central do Brasil, região essa que parece não ter importância como ponte entre a Floresta Amazônica e a Mata Atlântica. A mesma autora afirma que o sudeste da floresta Amazônica é mais próximo filogeograficamente do norte da Mata Atlântica. A parte da floresta Amazônica, onde E. (A.) sericea é mais frequentemente coletada, parece estar mais próxima filogeograficamente da parte da Mata Atlântica que engloba o estado do Espírito Santo.

Por fim, são necessários estudos para verificar a estruturação entre as populações de $E$. (A.) sericea da região Amazônica e a encontrada na região Sudeste já que o isolamento por distância pode ter restringido o fluxo gênico e essas populações podem estar sofrendo processo de especiação.

\section{REFERÊNCIAS}

Aguiar, G. M. \& W. M. Medeiros. 2003. Distribuição regional de habitats das espécies de flebotomíneos do Brasil. p. 207-256. In: Rangel E. F. \& R. Lainson (Org.) Flebotomíneos do Brasil. Rio de Janeiro: Fiocruz, iii+367 p.

Barretto, M. P. \& J. O. Coutinho. 1940. Processos de captura, dissecação e montagem de flebótomos. Annais da Faculdade de Medicina de São Paulo 16: 173-187.

Barros, G. C.; P. A. Sessa; E. A. Mattos; V. R. D. Carias; W. Mayrink; J. T. A. Alencar; A. Falqueto \& A. C. Jesus. 1985. Foco de leishmaniose tegumentar americana nos municípios de Viana e Cariacica, Estado do Espírito Santo, Brasil. Revista de Saúde Pública 19: 146153.

Costa, L. P. 2003. The historical bridge between the Amazon and the Atlantic Forest of Brazil: a study of molecular phylogeography with small mammals. Journal of Biogeography 30: 71-86.

Falqueto, A.; J. R. Coura; G. C. Barros; G. Grimaldi Junior; P. A. Sessa; V. R. D. Carias; A. C. Jesus \& J. T. A. Alencar. 1986. Participação do cão no ciclo de transmissão da leishmaniose tegumentar no município de Viana, Estado do Espírito Santo. Memórias do Instituto Oswaldo Cruz 81: 155-163.

Feitoza, L. R. 1999. Carta Agroclimática do Espírito Santo. Governo do Estado do Espírito Santo: Secretaria de Estado da Agricultura \& Empresa Capixaba de Pesquisa Agropecuária EMCAPA.

Ferreira, A. L.; P. A. Sessa; J. B. M. Varejão \& A. Falqueto. 2001. Distribution of sand flies (Diptera: Psychodidae) at different altitudes in an endemic region of American cutaneous leishmaniasis in the state of Espírito Santo, Brazil. Memórias do Instituto Oswaldo Cruz 96: 1061-1067.

Freitas, R. A.; R. D. Naiff \& T. V. Barret. 2002. Species diversity and flagellate infections in the sand fly fauna near Porto Grande, state of Amapá, Brazil (Diptera: Psychodidae. Kinetoplastida: Trypanosomatidae). Memórias do Instituto Oswaldo Cruz 97: 53-59.

Galati, E. A. B. 1995. Phylogenetic systematics of Phlebotominae 
(Diptera, Psychodidae) with emphasis on American groups. Boletín de la Dirección de Malariología y Saneamento Ambiental 35: 133-142.

Galati, E. A. B. 2003a. Classificação de Phlebotominae. p. 23-51 In: Rangel E. F. \& R. Lainson (Org.). Flebotomíneos do Brasil. Rio de Janeiro: Fiocruz, ii+367 p.

Galati, E. A. B. 2003b. Morfologia, terminologia de adultos e identificação dos táxons da América. p. 53-175. In: Rangel E. F. \& R. Lainson (Org.). Flebotomíneos do Brasil. Rio de Janeiro: Fiocruz, ii+367 p.

Galati, E. A. B.; Marassá, A. M. \& R. M. G. Andrade. 2003. Micropygomyia (Sauromyia) petari, a new species of Phlebotominae (Diptera, Psychodidae) from Vale do Ribeira, São Paulo State, Brazil. Revista Brasileira de Entomologia 47: 455459.

Gebre-Michael, T.; J. B. Malone; M. Balkew; A. Ali; N. Berhe; A. Hailu \& A. A. Herzi. 2004. Mapping the potential distribution of Phlebotomus martini and P. orientalis (Diptera: Psychodidae), vectors of kala-azar in East Africa by use of geographic information systems Acta Tropica 90: 73-86.

Martins, A. V.; P. Williams \& A. L. Falcão. 1978. American sand flies (Diptera: Psychodidae, Phlebotominae). Academia Brasileira de Ciências, 195 p.
Peterson, A. T. \& J. Shaw. 2003. Lutzomyia vectors for cutaneous leishmaniasis in Southern Brazil: ecological niche models, predicted geographic distributions, and climate change effects. International Journal of Parasitology 33: 919-931.

Pinto, I. S. \& C. B. Santos. 2007. Description of Lutzomyia (Lutzomyia) falquetoi sp. nov. (Diptera: Psychodidae, Phlebotominae) a new species from the state of Espírito Santo, Brazil. Memórias Instituto Oswaldo Cruz 102: 165-167.

Pinto, I. S.; J. G. Pires; C. B. Santos; T. M. Virgens; G. R. Leite; A. L. Ferreira \& A. Falqueto. 2008. Primeiro registro de Nyssomyia yuilli yuilli (Young \& Porter) e de Trichopygomyia longispina (Mangabeira) (Diptera: Psychodidae) no estado do Espírito Santo, Brasil. Biota Neotropica 8: 221-223.

Shimabukuro, P. H. F.; A. M. Marassá \& E. A. B. Galati. 2007. Brumptomyia carvalheiroi sp. nov. (Diptera: Psychodidae: Phlebotominae) from Atlantic forest domain, São Paulo State, Brazil. Zootaxa 1637: 47-54.

Young, D. G. \& M. A. Duncan. 1994. Guide to the identification and geographic distribution of Lutzomyia sand flies in Mexico, the West Indies, Central and South America (Diptera: Psychodidae). Memoirs of the American Entomological Institute 54, Associated Publishers, $881 \mathrm{p}$. 